\title{
High frequency generation in the corona: Resonant cavities
}

\author{
I. C. Santamaria ${ }^{1,2}$ and T. Van Doorsselaere ${ }^{1}$ \\ ${ }^{1}$ Centre for Mathematical Plasma Astrophysics, Department of Mathematics, KU Leuven, Celestijnenlaan 200B, 3001 Leuven, \\ Belgium \\ e-mail: irantzu.calvosantamaria@kuleuven.be \\ 2 Instituto de Astrofísica de Canarias, 38205 La Laguna, Tenerife, Spain
}

Received 21 April 2017 / Accepted 17 December 2017

\begin{abstract}
Aims. Null points are prominent magnetic field singularities in which the magnetic field strength strongly decreases in very small spatial scales. Around null points, predicted to be ubiquitous in the solar chromosphere and corona, the wave behavior changes considerably. Null points are also responsible for driving very energetic phenomena, and for contributing to chromospheric and coronal heating. In previous works we demonstrated that slow magneto-acoustic shock waves were generated in the chromosphere propagate through the null point, thereby producing a train of secondary shocks escaping along the field lines. A particular combination of the shock wave speeds generates waves at a frequency of $80 \mathrm{MHz}$. The present work aims to investigate this high frequency region around a coronal null point to give a plausible explanation to its generation at that particular frequency.

Methods. We carried out a set of two-dimensional numerical simulations of wave propagation in the neighborhood of a null point located in the corona. We varied both the amplitude of the driver and the atmospheric properties to investigate the sensitivity of the high frequency waves to these parameters.

Results. We demonstrate that the wave frequency is sensitive to the atmospheric parameters in the corona, but it is independent of the strength of the driver. Thus, the null point behaves as a resonant cavity generating waves at specific frequencies that depend on the background equilibrium model. Moreover, we conclude that the high frequency wave train generated at the null point is not necessarily a result of the interaction between the null point and a shock wave. This wave train can be also developed by the interaction between the null point and fast acoustic-like magneto-acoustic waves, that is, this interaction within the linear regime.
\end{abstract}

Key words. Sun: atmosphere - Sun: corona - Sun: magnetic fields - methods: numerical - magnetohydrodynamics (MHD) - waves

\section{Introduction}

Null points are magnetic field singularities where the magnetic field strength drops to zero in extremely small spatial scales. Because of their small sizes, a very high spatial resolution is needed to observe null points directly, which is not possible with the current instrumentation. Moreover, coronal magnetic fields are still hard to observe (Lin et al. 2004; Van Doorsselaere et al. 2008; Gibson et al. 2016), which makes it even more complicated. Nevertheless, the extrapolation of photospheric magnetic fields predict that null points are omnipresent in the solar chromosphere and corona (Galsgaard \& Nordlund 1997; Longcope 2005).

Although the null points are small magnetic structures, their presence in the solar atmosphere drastically changes the behavior of waves and flows around it (McLaughlin et al. 2011; Santamaria et al. 2015). Since the magnetic field is zero, the Alfvén speed is zero, and therefore magnetic waves cannot propagate through the magnetic field. In fact, these waves are refracted at the vicinity of null points owing to the gradient in the Alfvén velocity (Nakariakov \& Roberts 1995; McLaughlin \& Hood 2004; Santamaria et al. 2015). The only waves that can physically cross null points are acoustic nature waves. Besides the considerable change in magnetohydrodynamic waves behavior, null points can also drive highly energetic phenomena such as solar flares (Shibata \& Magara 2011). Also reconnection events are likely to take place in null points, dissipating energy into heat. Moreover, McLaughlin \& Hood (2004) showed an accumulation of current at the null point, which results in an increasing importance of dissipative processes that convert wave energy into heat. Hence, null points can also contribute to chromospheric and coronal heating.

Santamaria et al. (2016, 2017) have observed a high frequency wave train being developed in their two-dimensional null point. This phenomenon is pressure driven and is the result of the interaction between a slow acoustic-like magneto-acoustic shock wave developed in the chromosphere and a coronal null point. Moreover the wave train has a frequency of $80 \mathrm{MHz}$. This produces a high frequency region around the null point that dominates in the whole simulation run. Since, as mentioned above, null points are predicted to be everywhere in the solar chromosphere and corona, and the slow magneto-acoustic waves can easily steepen into shocks, the authors stated that this phenomenon might be very common in the solar atmosphere. They suggested that the short-period oscillations (between 8 and $12 \mathrm{~s}$ ) observed during the decay phase of flares (Melnikov et al. 2005; Van Doorsselaere et al. 2011, 2016) could also be interpreted as slow magneto-acoustic shock waves. Similar wave trains have been found in other numerical simulations (McLaughlin et al. 2009; Tarr et al. 2017), although the driving mechanisms in the cited works are different. However the authors of these works do not give any information about the periodicity and nature of the phenomenon, and hence we cannot compare these findings on the wave train frequencies. Other studies model the quasiperiodic pulsations(QPPs) representing their development in cusp-like magnetic field structures (Jakimiec \& Tomczak 2010; 
Takasao et al. 2015; Takasao \& Shibata 2016). In the numerical models presented in those works, shock waves are also generated, but again, both the generation and nature of these shock waves are different.

The present work aims to carry out an in-depth analysis of the high frequency region around the null point. To that aim we perform a set of numerical simulations of wave propagation around a two-dimensional null point. In order to investigate the origin of the high frequencies, we vary the amplitude of the driver to study how this affects the frequency distribution. On the other hand, we modify the properties of the atmospheric background model. This allows us to understand the mechanism of the generation of the high frequency waves around the null point.

\section{Description of the simulations}

We numerically solved the ideal nonlinear two-dimensional magnetohydrodynamic equations of conservation of mass, momentum, energy, and the induction equation for the magnetic field as follows:

$$
\frac{\partial \rho}{\partial t}+\nabla(\rho \mathbf{v})=\left[\frac{\partial \rho}{\partial t}\right]_{\mathrm{diff}},
$$

$$
\frac{\partial(\rho \mathbf{v})}{\partial t}+\nabla\left[\rho \mathbf{v} \mathbf{v}+\left(p+\frac{\mathbf{B}^{2}}{2 \mu_{0}}\right) \mathbf{I}-\frac{\mathbf{B B}}{\mu_{0}}\right]=\rho \mathbf{g}+\left[\frac{\partial(\rho \mathbf{v})}{\partial t}\right]_{\mathrm{diff}},
$$

$\frac{\partial p}{\partial t}+\mathbf{v} \nabla p+\gamma p \nabla \mathbf{v}=\left[\frac{\partial p}{\partial t}\right]_{\mathrm{diff}}$,

$$
\frac{\partial \mathbf{B}}{\partial t}=\nabla \times(\mathbf{v} \times \mathbf{B})+\left[\frac{\partial \mathbf{B}}{\partial t}\right]_{\mathrm{diff}},
$$

where all the notations are standard. We used an ideal equation of state with an adiabatic index of $\gamma=5 / 3$. The equations are solved by means of the code MANCHA (see Khomenko \& Collados 2006; Felipe et al. 2010, for details). The grid of the simulation domain is homogeneous with spatial resolutions of $\Delta x=50 \mathrm{~km}$ and $\Delta z=10 \mathrm{~km}$ along the horizontal and vertical directions, respectively. The spatial resolution is high enough to spatially resolve the $80 \mathrm{MHz}$ frequencies. The physical diffusive terms are replaced by their artificial analogous, similar to other numerical codes (Nordlund \& Stein 1990; Vögler et al. 2005; Gudiksen et al. 2011). These are the so-called hyperdiffusivities, required for the numerical stability of the code (terms with subscript "diff" in the equations). After extracting the equilibrium atmosphere (see below) from the equations, the evolution of the perturbations is computed.

To calculate the background equilibrium model we considered a potential magnetic field, and therefore we calculated the hydrostatic and magnetostatic models separately. The hydrostatic model is chosen to be plane-parallel and vertically stratified. In this work we focused on a small region around a coronal null point, in which we consider an isothermal plasma of $1 \mathrm{MK}$. From the temperature profile we calculated the density and pressure distributions by means of the hydrostatic equations. The magnetic field structure is potential and it is composed by two vertical flux tubes of the same polarity connected by arcadeshaped magnetic field structures (see field lines in left hand panel of Fig. 1). This magnetic field topology leads to a null point

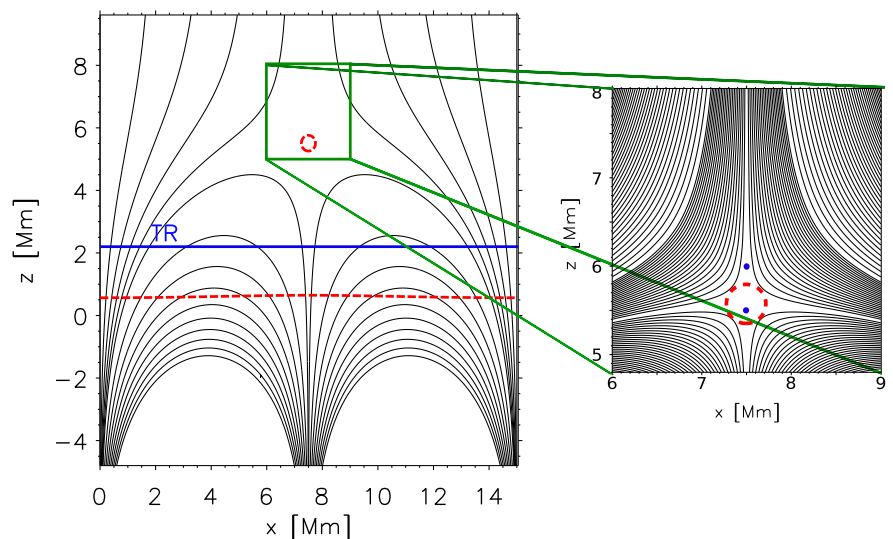

Fig. 1. Magnetic field lines for the entire simulation domain $(z=[-5,10] \mathrm{mm})($ left $)$ and the enhanced region around the null point (right). The red dashed curves show the equipartition layers and the horizontal blue line indicates the location of the transition region. The two blue dots in the right hand panel indicate the selected points for the measurement of the Alfvén and sound speeds in the sections below.

in the corona, which is the region under study in the present work (see right hand panel of Fig. 1). We selected the mentioned region around the null point, and we ensured that the magnetic field is divergence free and horizontally periodic.

In order to drive waves, we perturbed the null point by exerting an instantaneous pressure pulse below this null point written as

$\frac{\delta p_{1}}{p_{0}}=A \gamma \exp \left[-\left(\frac{\left(x-x_{0}\right)^{2}}{2 \sigma_{x}^{2}}+\frac{\left(z-z_{0}\right)^{2}}{2 \sigma_{z}^{2}}\right)\right]$,

$\frac{\delta \rho_{1}}{\rho_{0}}=A \exp \left[-\left(\frac{\left(x-x_{0}\right)^{2}}{2 \sigma_{x}^{2}}+\frac{\left(z-z_{0}\right)^{2}}{2 \sigma_{z}^{2}}\right)\right]$,

where $A$ is the amplitude of the pulse relative to the equilibrium variables. $\left[x_{0}, z_{0}\right]=[7500,5000] \mathrm{km}$ is the location of the center of the pulse, and $\sigma_{x}$ and $\sigma_{z}$ are the widths of the pulse in the horizontal and vertical directions, respectively. In order to carry out this study, we performed four numerical simulations for different pulse amplitudes, that is, for $A=0.5,0.9,1.5$, and 2. By choosing these amplitudes relative to the equilibrium variables, we made sure that the desired nonlinear regime is fulfilled.

Additional tests were carried out in order to make sure that the obtained results do not depend on the particular hyperdiffusion chosen. These tests show that the effect of the hyperdiffusive terms do not influence the results significantly.

\section{Wave behavior}

Figure 2 shows the evolution of the vertical velocity of the waves driven by the pressure pulse with $A=0.9$. The pulse expands and the fast magneto-acoustic wave front rapidly reaches the null point $(t=5.5 \mathrm{~s})$. As can be appreciated in the same panel, most of the fast magneto-acoustic waves getting close to the null point are refracted owing to the large gradient in the Alfvén speed. Few waves reach the equipartition contour surrounding the null point (black dashed contour) and experience partial conversion into fast magneto-acoustic waves ${ }^{1}$. A few seconds after the fast

1 We note that the plasma $\beta$ outside the $\beta=1$ contour around the null point is lower than one, while the plasma $\beta$ inside the contour is higher than one. Therefore the fast waves outside the equipartition contour are of magnetic nature and fast waves inside the equipartition contour are of acoustic nature. 

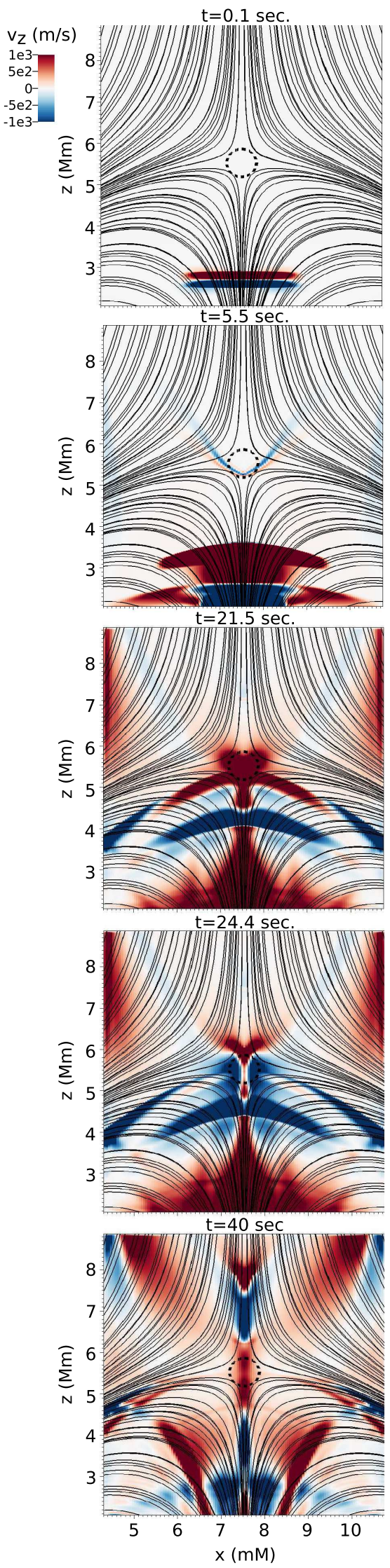

Fig. 2. Evolution of the vertical velocity for waves driven by an instantaneous pressure pulse in the neighborhood of a coronal null point. The amplitude of the pulse is $A=0.9$. The black solid lines show the magnetic field lines and the black dashed line indicate the equipartition layer where the plasma $\beta=1$. The simulation time is given above each panel in seconds.

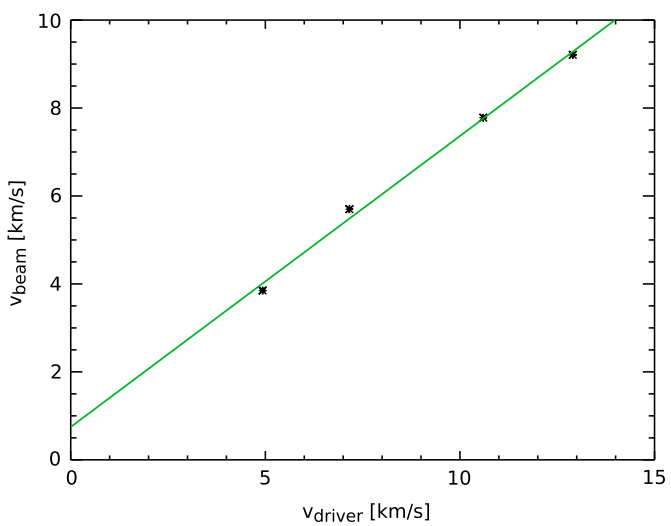

Fig. 3. Velocity amplitudes for the driven waves against the velocity amplitude of the waves developed at the null point for waves driven by the different amplitude drivers $A=0.5,0.9,1.5$, and 2 . The green line indicates the line fit of the distribution of points.

front, the slow front reaches the null point $(t=21.5 \mathrm{~s})$. The slow magneto-acoustic waves get to the equipartition contour and are partially transmitted into fast magneto-acoustic waves. The fast magneto-acoustic waves in the high $\beta$ region around the null point are the only waves that can physically cross the null. When the strong slow magneto-acoustic front interacts with the null point, it results in a train of waves that are strongly emitted by the null point. This is shown as a high frequency wave train propagating outward from the null point with supersonic velocity $\left(\mathrm{v}_{w t}\right.$ $\approx 128 \mathrm{~km} \mathrm{~s}^{-1}$ ).

Santamaria et al. (2017) have shown the same phenomena taking place in their two-dimensional simulation. In their work the wave train was the result of the interaction between the null point and a fast magneto-acoustic shock wave in the high $\beta$ plasma at the null. On the contrary, in Santamaria et al. (2015) where the same numerical simulation is shown but considering the linear regime of propagation, this behavior around the null point is not observed. In the present work we are in the nonlinear regime of wave propagation but there are no shock waves being developed. In order to analyze the linear or nonlinear nature of the wave train generation, we plot the velocity of the driver for the four different driving amplitudes against the velocity amplitude of the resulting wave train. Figure 3 shows that the obtained points have a linear dependence, which suggests that the wave train is a linear phenomenon and, therefore, it can be developed in both linear or nonlinear systems. As mentioned before in the linear numerical simulations of Santamaria et al. (2015), the wave train is not developed, and it might be due to the low amplitude of the driver in that simulation $\left(A=10^{-5}\right)$. On the other hand, Gruszecki et al. (2011) demonstrated that the nonlinear wave behavior around a two-dimensional null point differs considerably from the linear wave propagation.

\section{Frequency distribution}

In order to analyze the frequency of the wave train, we calculated the dominant frequency at each point of the domain. We repeated this calculation for the waves driven by the pulses with the four different amplitudes. Figure 4 shows the spatial distribution of the dominant frequencies for the four simulations carried out. It can be clearly appreciated that the pattern of the frequency distributions is very similar in all the cases. The frequency range around the null point and at the location of the wave train is $60-80 \mathrm{MHz}$ for all the simulation runs. These frequencies are approximately the same as those obtained by 

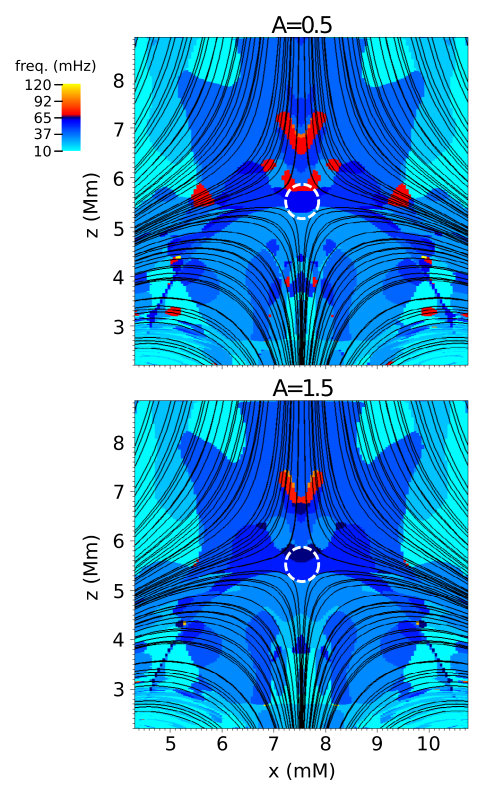

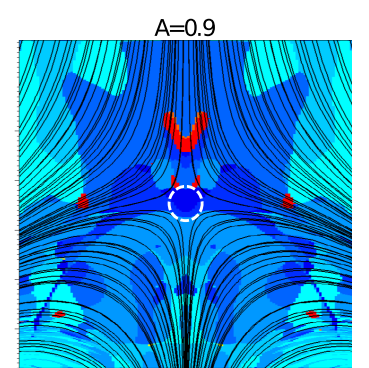

$A=2$

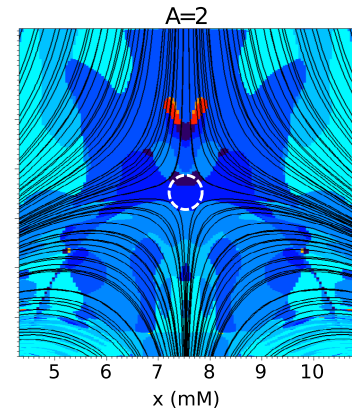

Fig. 4. Spatial distribution of the dominant frequencies for waves driven by the different amplitude drivers $A=0.5$ (top left-hand panel), $A=0.9$ (top right-hand panel), $A=1.5$ (bottom left-hand panel), and $A=2$ (bottom right-hand panel). The vertical velocity was used to calculate the Fourier transform in all the cases. The black solid lines indicate the magnetic field lines and the white dashed line shows the equipartition contour.
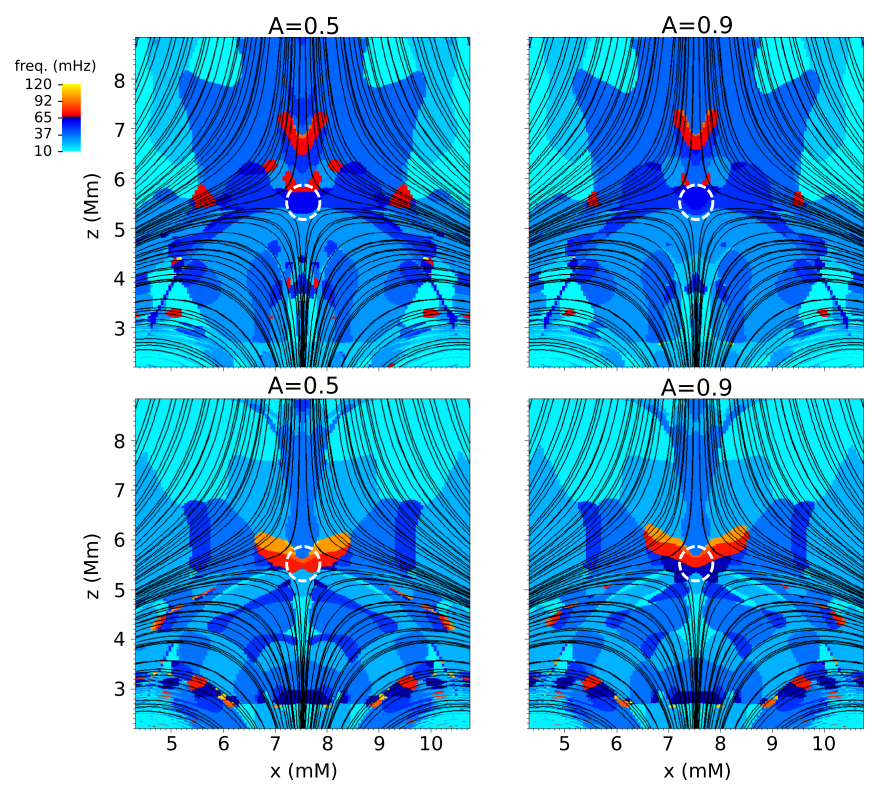

Fig. 5. Comparison of the spatial distribution of the dominant frequencies for waves propagating in two different background atmospheres driven by the different amplitude drivers $A=0.5$ and $\rho_{0}$ (top left-hand panel), $A=0.9$ and $\rho_{0}$ (top right-hand panel), $A=0.5$ and $\rho_{0}^{\prime}=\rho_{0} / 2$ (bottom left-hand panel), and $A=0.9$ and $\rho_{0}^{\prime}=\rho_{0} / 2$ (bottom right-hand panel). The vertical velocity was used to calculate the Fourier transform in all the cases. The black solid lines indicate the magnetic field lines and the white dashed line shows the equipartition contour.

Santamaria et al. (2017) where the region around the null point had a frequency of $80 \mathrm{MHz}$.

We speculated that the frequency distribution can be sensitive to the atmospheric properties. To investigate this dependency we halved the background density, that is, $\rho_{0}^{\prime}=\rho_{0} / 2$, where $\rho_{0}^{\prime}$ is the new equilibrium density, and we perturbed the new atmosphere by exciting pressure pulses with the same relative amplitudes as those in the previous simulations set up. Figure 5 shows the spatial distribution of the dominant frequencies for the previous case with the full background density $\rho_{0}$ (top panels) and the dominant frequencies for the new numerical setup with half of the background density (bottom panels). The right- and left-hand panels show the simulation runs with $A=0.5$ and $A=0.9$, respectively. It can be clearly seen how the frequency distribution around the null point changes with different background models.

This suggests to us that the null point is acting as a resonator, thereby producing those particular frequencies. Therefore, the frequency of the wave train depends on the atmospheric properties. This is very interesting since we could infer information about the atmosphere by detecting high frequency waves.

For a better visualization of the previous statement we calculated the spatial distribution of 10 different frequencies within the numerical domain. Figure 6 shows the spatial distributions of four of those frequencies: 20, 60, 70, and $90 \mathrm{MHz}$. It can be appreciated from the figure that although all the frequencies reach the null point, just the frequencies between $60-80 \mathrm{MHz}$ are channeled outward by the null point along the separatrixes. This suggests that the null point is acting as a resonator for those particular frequencies. To rule out that the high frequencies are artificially generated by the null point, we make the discontinuity smaller by increasing the spatial resolution. This way the large gradient in magnetic field is resolved more accurately. We performed an extra simulation with double resolution of the original one, keeping all the parameters identical to the original simulation. The spatial distribution of the dominant frequencies obtained is shown in Fig. 7. This demonstrates that the frequency distribution is independent from the grid resolution since both patterns are almost identical, and therefore the wave train is not artificially generated by a discontinuity at the null point. In order to check which quantities are responsible for the change in the frequency pattern, we performed two sets of numerical simulations in which the background Alfvén or sound speeds are changed. The first set of simulations consists of five numerical simulations in which the background sound speed has been changed and the second set of simulations is composed by four numerical simulations in which we varied the Alfvén speed. In order to check the frequency pattern modification we selected two points: one inside the high $\beta$ region around the null point and another in the low $\beta$ region close to the null. We compared the frequencies obtained at those points with the corresponding Alfvén and sound speeds. Figure 8 shows how the frequencies change with the variation of Alfvén and sound speeds, both inside and outside the high $\beta$ region around the null point. Overplotted are the obtained linear fits for the set of points. It can be seen that for both Alfvén and sound speed variations, we obtain a linear variation of the frequencies. Nevertheless, for the variation in the Alfvén speed a better fit of the points is obtained, which suggests that the null point is behaving as a magnetic resonant cavity (Craig \& McClymont 1991; Bulanov et al. 1992). Additionally, it is expected that the size of the cavity also changes the frequency distribution. Figure 9 shows the variation of the frequencies with the diameter of the $\beta=1$ contour surrounding the null points, which we consider to represent our resonant cavity. The plot shows that there is not a clear relationship between the shape of the equipartition contour and the frequencies obtained. This indicates that the equipartition layer does not represent the shape of the resonator. Since we have more than one frequency 

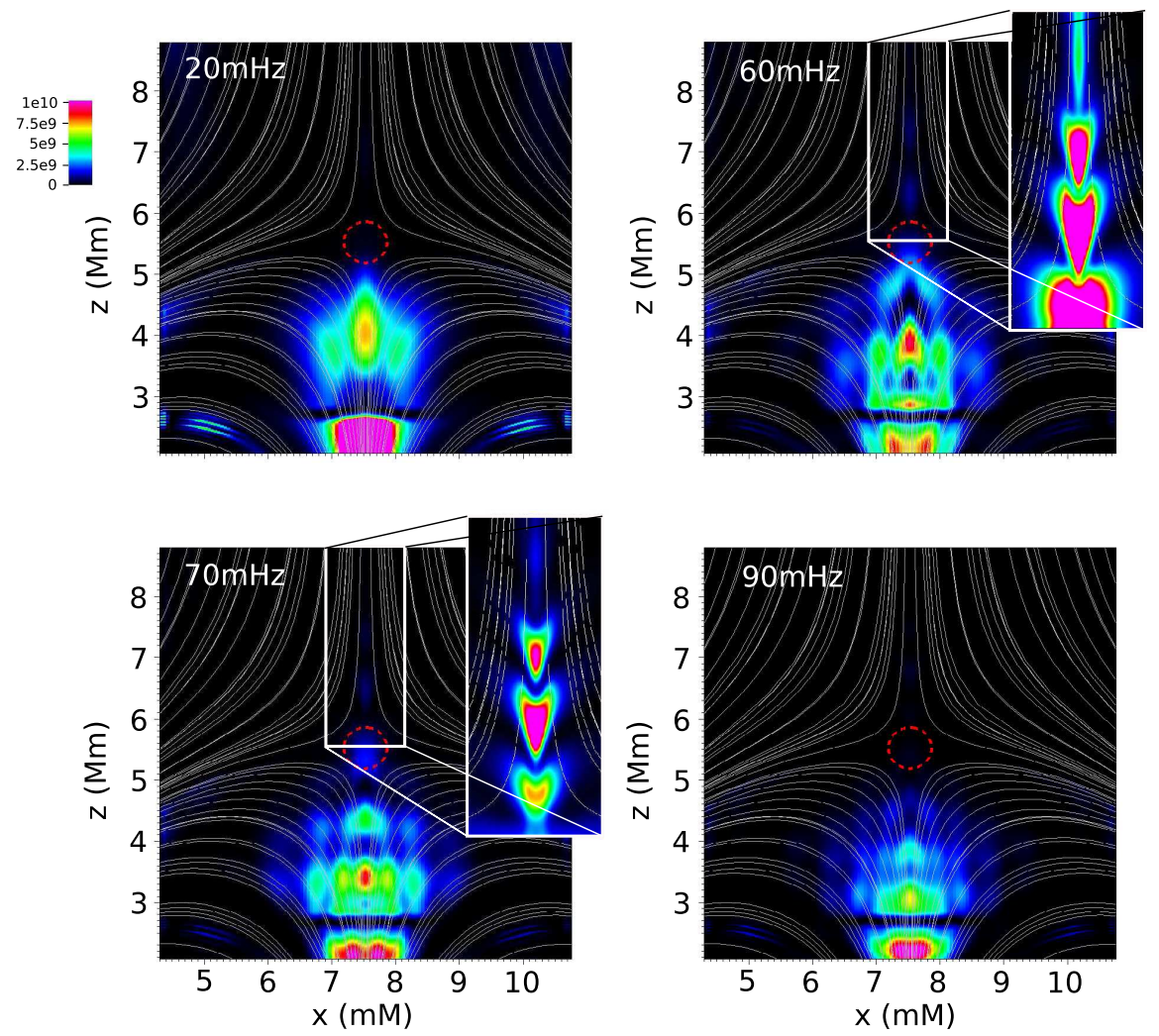

Fig. 6. Spatial distributions of four different frequencies. The color map shows the frequency power. The overplotted light gray lines indicate the magnetic field lines and the dashed red contour shows the equipartition layer around the null point. The wave train was saturated in the prominent cases for a better visualization.
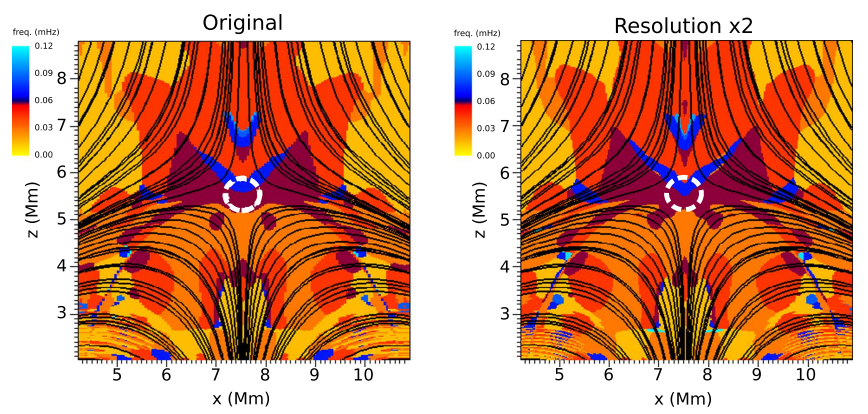

Fig. 7. Spatial distributions of the dominant frequencies for two different grid resolutions: (left) the original simulation with $351 \times 700$ grid points and (right) simulation with double resolution $(700 \times 1400$ grid points). The color map shows the frequency power. The overplotted black lines indicate the magnetic field lines and the dashed white contour shows the equipartition layer around the null point.

channeled along the separatrixes by the null point, we suspect that there is not a single resonant cavity, but a resonator per generated frequency. Nevertheless, and having checked the results deeply, we consider that it is not feasible to give the exact size or shape of the resonators. This is shown clearly in the insets of Fig. 6, where the shape of the resonator is unclear because it is superposed with the initial pulse profile and beam wave energy.

\section{Discussion and conclusions}

In the present work we extend the analysis of Santamaria et al. (2017) who have found high frequency regions generated around coronal null points. Concretely, we focus on the possible sensitivity of these frequencies to the background atmospheric properties. To that aim we carried out a set of numerical simulations of wave propagation around a two-dimensional null point located in the solar corona. The waves are excited by an
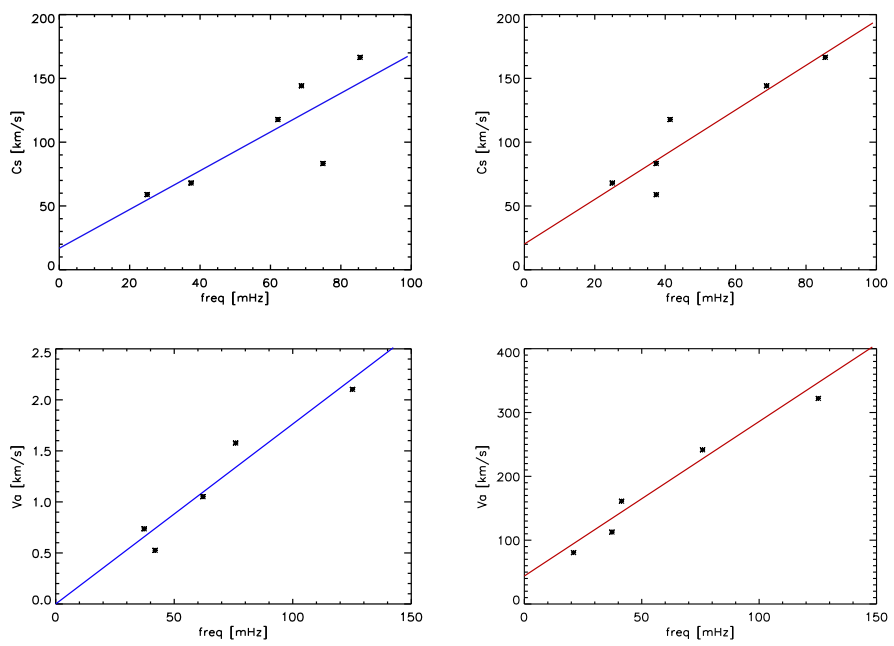

Fig. 8. Frequency dependence with the sound speed (top panels) and Alfvén speed (bottom panels) for the sets of simulations carried out with different Alfvén and sound speeds. The overplotted lines are the linear fits of the points obtained from the simulations. The left-hand panels correspond to a point inside the high $\beta$ region around the null point, while the right-hand panels correspond to a selected point in the low beta region close to the null point (see the blue dots in Fig. 1).

instantaneous pressure pulse excited just below the null point. In order to investigate the particular frequencies of $80 \mathrm{MHz}$, we changed the atmospheric properties of the background model within the set of numerical simulations. The interaction between fast magneto-acoustic waves and the null point leads to a high frequency wave train, which is in accordance with Santamaria et al. (2017).

In Santamaria et al. (2017) the authors have concluded that the jet-like feature is a train of secondary slow magneto-acoustic shock waves, that is the results of the interaction between an 


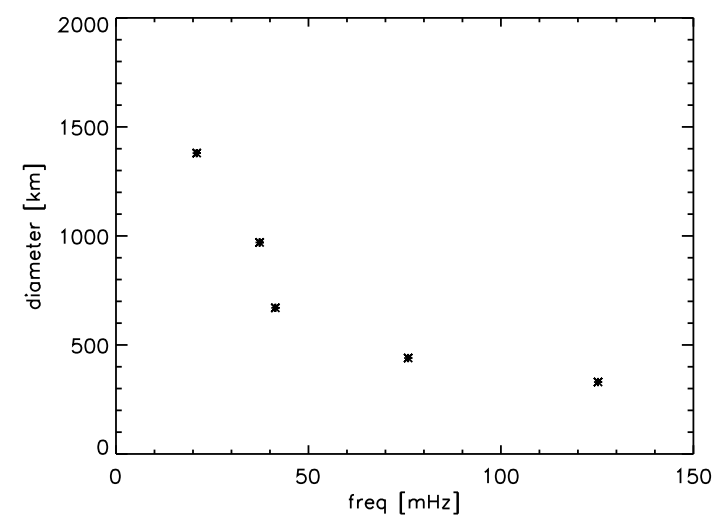

Fig. 9. Frequency dependence with the diameter of the cavity $(\beta=1$ contour) for the set of simulations carried out with different magnetic field strengths. The selected point is located in the low beta region close to the null point (see the blue dots in Fig. 1).

slow magneto-acoustic shock wave generated in the chromosphere and the null point. The wave train is pressure driven and the null point acts as a piston, driving waves strongly outward along the field lines. The frequency of this wave train is $80 \mathrm{MHz}$. In the current investigation we demonstrated that the wave train is also developed in the absence of shock waves. We have shown how an acoustic front interacts with the null point, generating the same sort of wave train. The train of waves is generated in all our simulations. On the other hand, Santamaria et al. (2015) showed that in the linear regime the train of waves is not generated. In the present work we demonstrated that the development of the high frequency wave train is a linear phenomenon. However we need the amplitude of the driver to be large enough for the wave train to be developed. In the present work, shocks are not developed but the amplitude of the driver is large enough. Moreover, the train of secondary waves have frequencies between 60 and $80 \mathrm{MHz}$, which is in accordance to those found by Santamaria et al. (2017).

We performed a set of four numerical simulations in which the only difference between the setups is the amplitude of the pulse. We found that the variation of the amplitude of driver does not alter the frequency distribution. This was also observed by Inglis et al. (2016). In their work they analyzed a large sample of QPP in solar flares using GOES and Fermi/GBM instruments. For both instruments, these authors have found that there is no relationship between the flare magnitude and the period of the event. Moreover, the distribution of periods found for the events are centered at $10-15 \mathrm{~s}$, which is consistent with the periods found in our numerical simulations (12.5-16.5 $\mathrm{s}$ approx).

We note that in Santamaria et al. (2017) the authors have speculated that the observed short-period waves in QPP (Nakariakov \& Melnikov 2009; Van Doorsselaere et al. 2016) could also be interpreted as slow magneto-acoustic shock waves, since the observed periods are very similar to those found around the null point in their numerical simulation. Additionally, since null points are theoretically predicted to be ubiquitous in the solar chromosphere and corona, and the slow magneto-acoustic waves can easily propagate into the corona, this phenomenon might be taking place frequently in the solar atmosphere. In fact, similar wave trains have been found in other works (Kliem et al. 2000; McLaughlin et al. 2009; Tarr et al. 2017), although they are driven by periodic reconnection processes.

In order to analyze the physical origin of the particular frequency distribution obtained in the first set of simulations, we changed the atmospheric background and we ran a new set of simulations using the same driving mechanism. With the comparison between the two sets of simulations, we observed that the frequency distribution changes with the atmospheric properties. This suggest that the particular frequencies observed around the null point are generated by resonance, and therefore the null point is behaving as a resonant cavity; the produced frequencies are subject to the thermodynamic structure of the atmosphere around the null point.

As a final step and with the aim of relating the atmospheric magnitudes with the obtained frequency distributions, we performed two sets of numerical simulations. In the first set, composed of five different simulations, we varied the sound speed and in the second set, which consisted of four different simulations, we changed the Alfvén speed. The obtained fits for the frequency dependence with the different velocities suggests that the null point is acting as a magnetic resonator. We also considered whether the frequency distributions depend on the size of the resonant cavity. Nevertheless, we were not able to demonstrate it in the present work. The cavity is superposed with the initial pulse profile and beam wave energy, and thus, the shape of the resonant cavity is unclear.

Acknowledgements. TVD was supported by an Odysseus grant of the FWO Vlaanderen, the IAP P7/08 CHARM (Belspo), and the GOA-2015-014 (KU Leuven).

\section{References}

Bulanov, S. V., Shasharina, S. G., \& Pegoraro, F. 1992, Plasma Phys. Controlled Fusion, 34, 33

Craig, I. J. D., \& McClymont, A. N. 1991, ApJ, 371, L41

Felipe, T., Khomenko, E., \& Collados, M. 2010, ApJ, 719, 357

Galsgaard, K., \& Nordlund, Å. 1997, J. Geophys. Res., 102, 231

Gibson, S., Kucera, T., \& White, S., et al. 2016, Frontiers in Astronomy and Space Sciences, 3, 8

Gruszecki, M., Vasheghani Farahani, S., Nakariakov, V. M., \& Arber, T. D. 2011, A\&A, 531, A63

Gudiksen, B. V., Carlsson, M., \& Hansteen, V. H., et al. 2011, A\&A, 531, A154 Inglis, A. R., Ireland, J., Dennis, B. R., Hayes, \& L., Gallagher, P. 2016, ApJ, 833,284

Jakimiec, J., \& Tomczak, M. 2010, Sol. Phys., 261, 233

Khomenko, E., \& Collados, M. 2006, ApJ, 653, 739

Kliem, B., Karlický, M., \& Benz, A. O. 2000, A\&A, 360, 715

Lin, H., Kuhn, J. R., \& Coulter, R. 2004, ApJ, 613, L177

Longcope, D. W. 2005, Liv. Rev. Sol. Phys., 2, 7

McLaughlin, J. A., \& Hood, A. W. 2004, A\&A, 420, 1129

McLaughlin, J. A., De Moortel, I., Hood, A. W., \& Brady, C. S. 2009, A\&A, 493, 227

McLaughlin, J. A., Hood, A. W., \& de Moortel, I. 2011, Space Sci. Rev., 158, 205

Melnikov, V. F., Reznikova, V. E., Shibasaki, K., \& Nakariakov, V. M. 2005, A\&A, 439, 727

Nakariakov, V. M., \& Melnikov, V. F. 2009, Space Sci. Rev., 149, 119

Nakariakov, V. M., \& Roberts, B. 1995, Sol. Phys., 159, 399

Nordlund, A., \& Stein, R. F. 1990, Comput. Phys. Commun., 59, 119

Santamaria, I. C., Khomenko, E., \& Collados, M. 2015, A\&A, 577, A70

Santamaria, I. C., Khomenko, E., Collados, M., \& de Vicente, A. 2016, A\&A, 590, L3

Santamaria, I. C., Khomenko, E., Collados, M., \& de Vicente, A. 2017, A\&A, 602, A43

Shibata, K., \& Magara, T. 2011, Liv. Rev. Sol. Phys., 8, 6

Takasao, S., \& Shibata, K. 2016, ApJ, 823, 150

Takasao, S., Matsumoto, T., Nakamura, N., \& Shibata, K. 2015, ApJ, 805, 135

Tarr, L. A., Linton, M., \& Leake, J. 2017, ApJ, 837, 94

Van Doorsselaere, T., Nakariakov, V. M., Young, P. R., \& Verwichte, E. 2008, A\&A, 487, L17

Van Doorsselaere, T., De Groof, A., Zender, J., Berghmans, D., \& Goossens, M. 2011, ApJ, 740, 90

Van Doorsselaere, T., Kupriyanova, E. G., \& Yuan, D. 2016, Sol. Phys., 291, 3143

Vögler, A., Shelyag, S., \& Schüssler, M., et al. 2005, A\&A, 429, 335 